\title{
Evaluation of gamma-ray strength function based on measured gamma-ray pulse-height spectra in time-of-flight neutron capture experiments
}

\author{
Nobuyuki Iwamoto ${ }^{1, *}$, Shoji Nakamura ${ }^{1}$, Atsushi Kimura ${ }^{1}$, Tatsuya Katabuchi ${ }^{2}$, Gerard Rovira $^{2}$, Kaoru, Y. Hara ${ }^{3}$, and \\ Osamu Iwamoto ${ }^{1}$ \\ ${ }^{1}$ Nuclear Data Center, Nuclear Science and Engineering Center, Japan Atomic Energy Agency, Tokai-mura, Naka-gun, Ibaraki 319- \\ 1195, Japan \\ ${ }^{2}$ Laboratory for Advanced Nuclear Energy, Tokyo Institute of Technology, Ookayama, Meguro-ku, Tokyo 152-8550, Japan \\ ${ }^{3}$ Faculty of Engineering, Hokkaido University, Sapporo, Hokkaido 060-8628, Japan
}

\begin{abstract}
In order to develop an evaluation method of gamma-ray strength function (GSF), neutron capture pulse-height $(\mathrm{PH})$ spectrum of gold was employed, where it was measured with the NaI(Tl) spectrometer of ANNRI installed at the Material and Life Science Experimental Facility in J-PARC. The neutron capture gammaray spectrum of gold was calculated using the nuclear reaction model code CCONE. In order to obtain the information on GSF from the measured data, a gamma-ray response function for the NaI(Tl) spectrometer was calculated by the Monte-Carlo particle-transport simulation code PHITS. As a result, the PH spectrum comparable with measured one was derived by applying the gamma-ray response function to the calculated gamma-ray spectrum. By evaluation with measured PH spectra, we obtained GSF which reasonably explains measured $\mathrm{PH}$ spectrum in the continuum region.
\end{abstract}

\section{Introduction}

Gamma-ray strength function (GSF) as well as nuclear level density is important ingredient to calculate neutron capture cross sections, since it provides energy-dependent transition strength of gamma-rays and directly relates to a spectrum of emitted gamma-rays. Therefore, an improvement of GSF is essential to enhance the reliability of neutron capture cross sections.

The shape of GSF has been derived by experiments of, for example, photo-induced reaction [1, 2] and $\left({ }^{3} \mathrm{He}^{3} \mathrm{He}^{\prime} \gamma\right)$ reaction $[3,4]$. In addition, the use of information on pulse-height $(\mathrm{PH})$ spectrum measured by neutron time-of-flight (TOF) experiments leads to an effective evaluation of GSF. The neutron TOF experiments have already been done with motivation to measure the gammaray spectra in the $\mathrm{keV}$ to $\mathrm{MeV}$ energy regions at various research institutes: ORNL [5, 6], Tokyo Tech. [7] and CEA [8] and so on.

The purpose of our research project entitled "Study on accuracy improvement of fast-neutron capture reaction data of long-lived minor actinides (MAs) for development of nuclear transmutation systems" is to accurately measure and derive the neutron capture cross sections of MAs in the keV neutron energy region. In this project, the neutron capture measurements are performed using the Accurate Neutron-Nucleus Reaction Measurement Instrument (ANNRI) [9] in Material and Life Science Experimental Facility (MLF) at J-PARC. The ANNRI has Ge and NaI(Tl)

*e-mail: iwamoto.nobuyuki@jaea.go.jp spectrometers that can record neutron TOF and $\mathrm{PH}$ spectra simultaneously. The latter spectrometer has the characteristic of rapid response which is suitable to measure cross sections in a fast-neutron energy region. This is why the data acquired by the $\mathrm{NaI}(\mathrm{Tl})$ spectrometer were used in the present work. In the measurement by the ANNRI, the PH spectra have been helpful to isolate resonances of impurity nuclides from the observed TOF spectra. Nevertheless, those have not been actively used to extract the information on GSF so far.

\section{Evaluation}

The present work is aimed to develop an evaluation method for GSF from PH spectrum. This method is different from previous analyses of GSF with neutron capture gamma-ray spectrum. This main reason is to reduce the uncertainty coming from the unfolding technique. Our approach is based on the folding of capture gamma-ray spectrum obtained by a nuclear reaction model, using gammaray response functions of the $\mathrm{NaI}(\mathrm{Tl})$ spectrometer. In either unfolding or folding method, the response function is a key component which should be reliably prepared.

\subsection{Response function of $\mathrm{Nal}(\mathrm{TI})$ spectrometer}

In order to derive a response function for the $\mathrm{NaI}(\mathrm{Tl})$ spectrometer, the Monte-Carlo particle-transport simulation code PHITS [10] was employed. The response of the spectrometer by monochromatic gamma-rays emitted at the sample position $(x, z)=(0,0)$ in Fig. 1 was calculated 
by the realistic simulated system [11] illustrated with top cross-sectional view in Fig. 1, in which two $\mathrm{NaI}(\mathrm{Tl})$ spectrometers are placed at 90 and 125 degrees with respect to the beam line. In the present analysis the detected data by larger spectrometer at 90 degree were adopted.

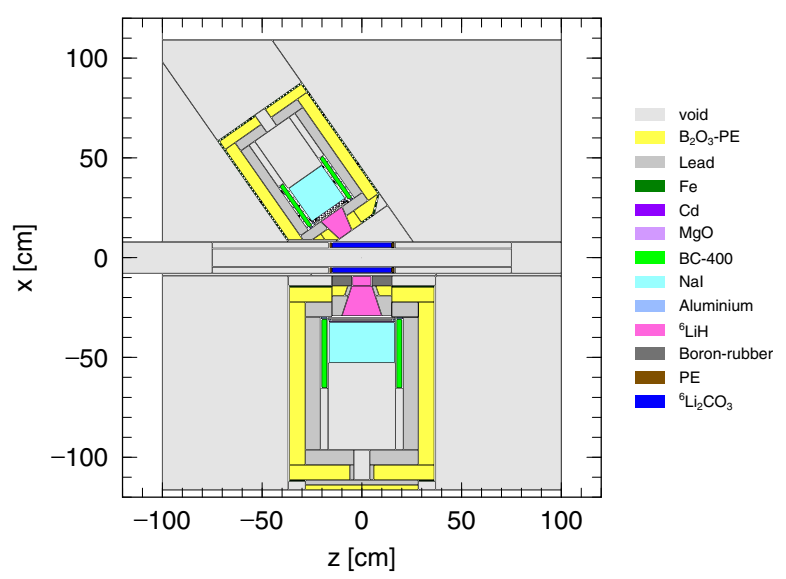

Figure 1. Simulated system (top cross-sectional view) of $\mathrm{NaI}(\mathrm{Tl})$ spectrometers in ANNRI. The pulsed neutron beam comes from negative direction of $z$-axis along the tube on $x=0$ line. Target is placed at $(x, z)=(0,0)$.

The evaluation of resolution in the spectrometer was made by comparing the simulated results with measured $\mathrm{PH}$ spectra of checking sources, ${ }^{60} \mathrm{Co},{ }^{137} \mathrm{Cs}$ and ${ }^{22} \mathrm{Na}$. The fitted results for three checking sources are shown in Fig. 2. The resolution in the energies higher than $1.3 \mathrm{MeV}$ was extrapolated on the basis of these results. The obtained response functions as a function of gamma-ray energy are presented in Fig. 3, in which the functions are prepared $100 \mathrm{keV}$ and $500 \mathrm{keV}$ each for incident gammaray energies lower and higher than $1 \mathrm{MeV}$, respectively. Though the present response functions are relative, the results are useful enough for evaluating the gamma-ray strength function.

\subsection{Neutron capture gamma-ray spectrum}

Hauser-Feshbach statistical model code CCONE [12] was used to calculate capture gamma-ray spectrum and evaluate the GSF. The physical ingredients related to low energy neutron reaction are mainly nuclear level density, discrete levels, neutron transmission coefficients (optical model) and GSF involved with E1, M1 and E2 transitions. The nuclear level density model was adopted from composite formulation by Gilbert \& Cameron [13], where the Fermi-gas model was updated with a revised level density parameter [14]. The information on discrete levels (i.e., excitation energy, spin, parity and gamma-ray transition probability and energy) was taken from RIPL-3 database of IAEA Nuclear Data Section [15]. Neutron transmission coefficient of ${ }^{197} \mathrm{Au}$ was calculated by the optical model with coupledchannels approach using three levels of ground state (spinparity $\left.J^{\pi}=3 / 2^{+}\right), 279 \mathrm{keV}\left(5 / 2^{+}\right)$and $548 \mathrm{keV}\left(7 / 2^{+}\right)$. The form of the optical model potential in the OPTMAN
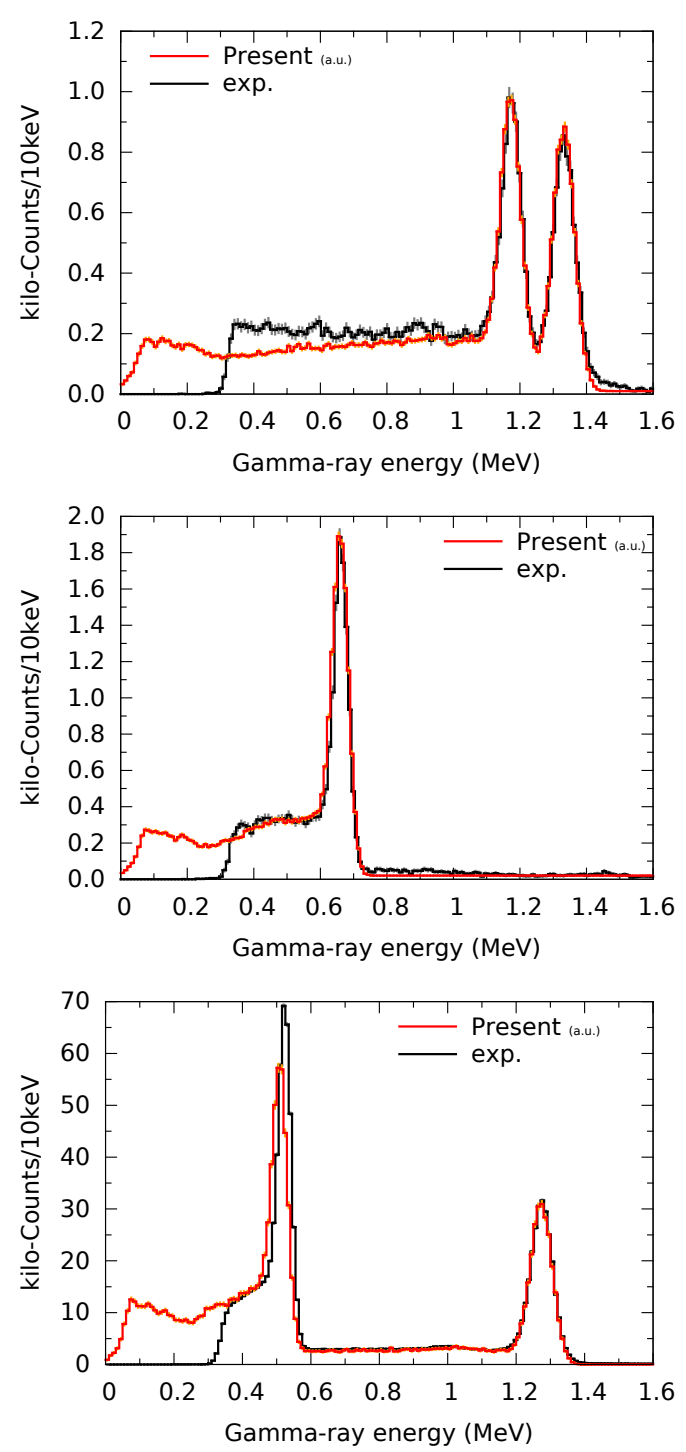

Figure 2. Comparisons of simulated PH spectra (scaled) with measured data for three checking sources, ${ }^{60} \mathrm{Co}$ (top), ${ }^{137} \mathrm{Cs}$ (middle) and ${ }^{22} \mathrm{Na}$ (bottom). There is subtle detection below $0.3 \mathrm{MeV}$ due to setting the discrimination level to the value.

code [16] was taken with optimized parameters. Gammaray transitions between continuum and continuum states and between continuum state and discrete level were calculated by E1, M1 and E2 GSFs. The GSF of standard Lorentzian form with energy-dependent damping width was applied for E1 transition [17]. The default parameters for E1 GSF were adopted from RIPL-2 [18]. The M1 and E2 transitions were calculated by the standard Lorentzian form [17].

\section{Results}

The capture gamma-ray spectrum was calculated assuming gamma-ray decay from an excited state with energy of $1 \mathrm{eV}$ and $J^{\pi}=2^{+}$in the compound nucleus ${ }^{198} \mathrm{Au}$. The excited state was presumed to be in a region with large contribution from the 4.9-eV $s$-wave resonance. The evaluation of dominant E1 GSF was performed by changing 


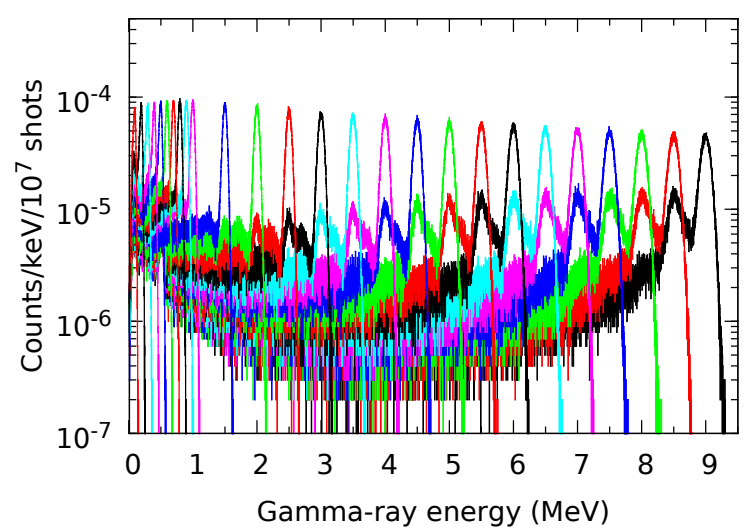

Figure 3. Gamma-ray response function obtained by the PHITS simulation for the $\mathrm{NaI}(\mathrm{Tl})$ spectrometer placed at 90 degrees with respect to the beam line.

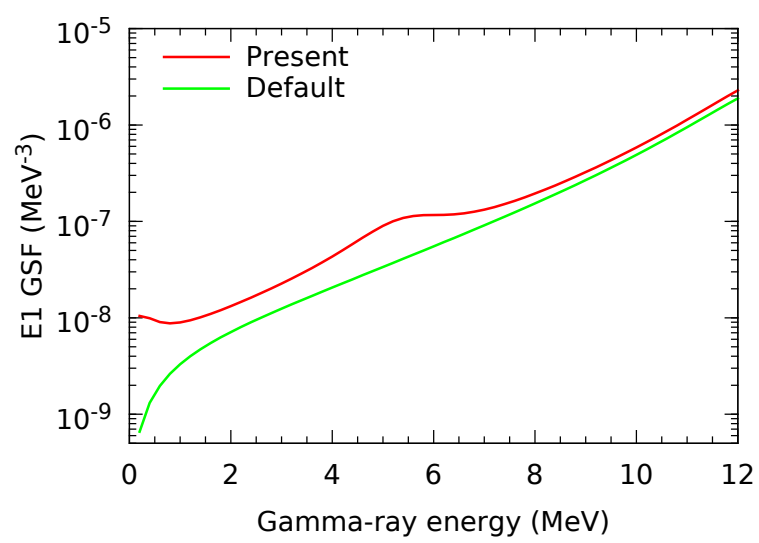

Figure 4. Comparison of the evaluated GSF for E1 transition from an excited state at $12 \mathrm{MeV}$ with that calculated by default E1 GSF parameters.

the parameters (i.e., resonance energy, resonance width and peak cross section) so as to reproduce measured $\mathrm{PH}$ spectrum. The evaluated result of E1 GSF for decay from $12 \mathrm{MeV}$ is shown in Fig. 4, in which the GSF calculated with default parameters is also presented. The capture gamma-ray spectra with the evaluated and default E1 GSFs are illustrated in Fig. 5. The gamma-ray response function was folded to the capture gamma-ray spectra. In order to do that, the neighboring response function with a higher incident gamma-ray energy was chosen. The gamma-ray energy in the response function was scaled by a factor of incident energy ratio above annihilation gamma-ray energy. More precisely, PHITS with the simulated system of spectrometer should be applied to the folding calculation. However, since it takes much time to obtain a folded result, this is not reasonable for an evaluation work. The obtained PH spectrum is shown in Fig. 6 together with the data of gold sample measured by the $\mathrm{NaI}(\mathrm{Tl})$ spectrometer in J-PARC MLF ANNRI for comparison purposes. In the continuum region of 0.5 to $5 \mathrm{MeV}$, the PH spectrum derived with evaluated GSF shows good

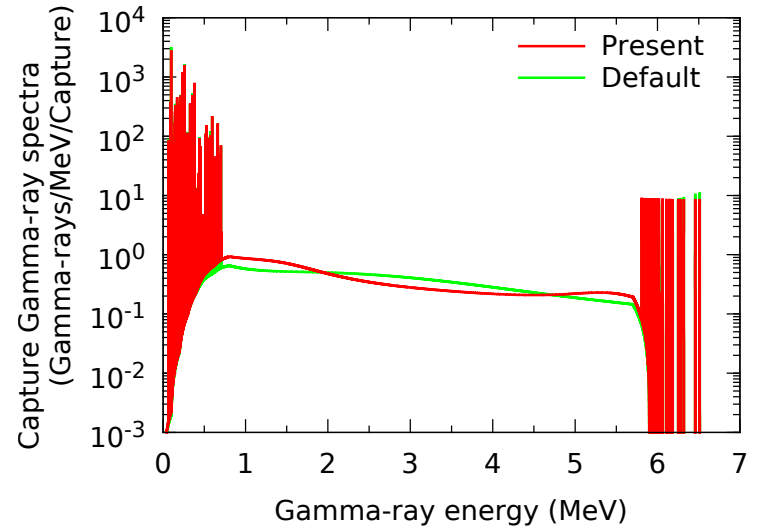

Figure 5. Comparison of the calculated capture gamma-ray spectrum of gold with default one for neutron incident energy of $1 \mathrm{eV}$. The transition gamma-rays from capture state to discrete levels and between discrete levels have sharp peaks.

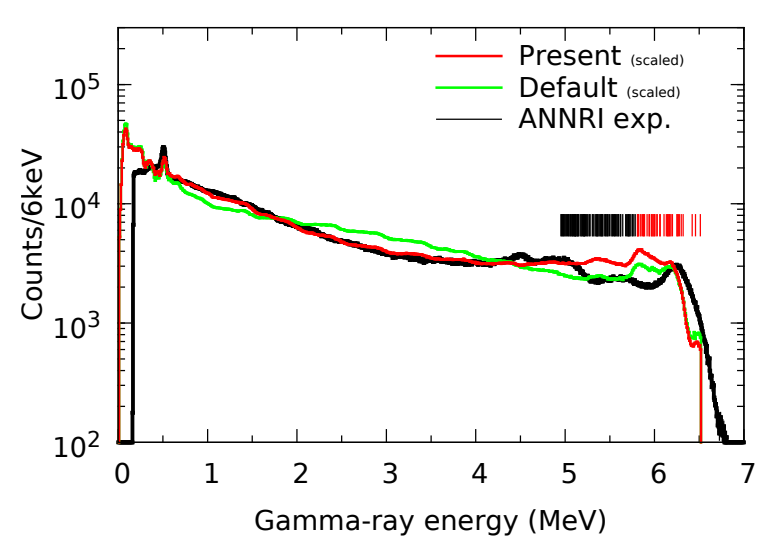

Figure 6. Comparison of the evaluated and default PH spectra of gold with measured data. Both results are scaled with a same factor so as to be matched with the measured data at the higher energy tail of the $511 \mathrm{keV}$ annihilation gamma-ray. The vertical lines at right middle part represent the known discrete levels of ${ }^{198} \mathrm{Au}$. The discrete levels adopted in the evaluation and not adopted are shown by red and black lines, respectively.

agreement with the measured data. On the other hand, in the spectrum above $5 \mathrm{MeV}$ the direct gamma-decay process from the capture state is dominant. The adjustments of transition probability to discrete levels might be needed. Note that the evaluated result was normalized to the count at higher energy tail of $511 \mathrm{keV}$ peak. In contrast, the $\mathrm{PH}$ spectrum calculated with default parameters is not matched with measured one, where the same normalization factor was applied here.

\section{Summary}

The PH spectrum of gold measured by the NaI(Tl) spectrometer of ANNRI installed in J-PARC MLF was used to develop a method for the evaluation of GSF. The neutron capture gamma-ray spectrum was calculated by using the Hauser-Feshbach statistical model code CCONE. The 
gamma-ray response function of the $\mathrm{NaI}(\mathrm{Tl})$ spectrometer was derived by the Monte-Carlo particle-transport simulation code PHITS with detailed simulated system of the spectrometer. Calculated capture gamma-ray spectrum was folded by the response function to generate $\mathrm{PH}$ spectrum. The evaluation of E1 GSF with sets of three parameters was performed to reproduce the measured $\mathrm{PH}$ spectrum. The obtained $\mathrm{PH}$ spectrum shows a reasonable agreement with the measured one in the continuum region where the contribution of GSF is strongly seen. It is confirmed that the evaluation method just introduced is usable to extract the information on GSF from the measured PH spectrum.

This work is supported by the Innovative Nuclear Research and Development Program from the Ministry of Education, Culture, Sports, Science and Technology of Japan.

The neutron experiment at the MLF of the J-PARC was performed under the user program (Proposal No. 2018B0011).

\section{References}

[1] R.R. Harvey, J.T. Caldwell, R.L. Bramblett, S.C. Fultz, Phys. Rev. 136, B126 (1964)

[2] A. Veyssiere, H. Beil, R. Bergere, P. Carlos, A. Lepretre, Nuclear Physics A 159, 561 (1970)

[3] A. Schiller, L. Bergholt, M. Guttormsen, E. Melby, J. Rekstad, S. Siem, Nuclear Instruments and Methods in Physics Research Section A: Accelerators, Spectrometers, Detectors and Associated Equipment 447, 498 (2000)

[4] N.U.H. Syed, M. Guttormsen, F. Ingebretsen, A.C. Larsen, T. Lönnroth, J. Rekstad, A. Schiller, S. Siem, A. Voinov, Phys. Rev. C 79, 024316 (2009)

[5] G.L. Morgan, E. Newman, Tech. rep., Oak Ridge National Laboratory (1975), ORNL-TM-4973

[6] J.K. Dickens, G.L. Morgan, G.T. Chapman, T.A. Love, E. Newman, F.G. Perey, Nuclear Science and Engineering 62, 515 (1977)
[7] M. Igashira, H. Kitazawa, M. Shimizu, H. Komano, N. Yamamuro, Nuclear Physics A 457, 301 (1986)

[8] J. Voignier, S. Joly, G. Grenier, Nuclear Science and Engineering 112, 87 (1992)

[9] M. Igashira, Y. Kiyanagi, M. Oshima, Nuclear Instruments and Methods in Physics Research Section A: Accelerators, Spectrometers, Detectors and Associated Equipment 600, 332 (2009)

[10] T. Sato, Y. Iwamoto, S. Hashimoto, T. Ogawa, T. Furuta, S. Abe, T. Kai, P.E. Tsai, N. Matsuda, H. Iwase et al., Journal of Nuclear Science and Technology 55, 684 (2018)

[11] K.Y. Hara, N. Fujii, T. Kamiyama, Y. Narita, H. Sato, S. Nakamura, Y. Toh, Measurement and Simulation of the Neutron Capture Reaction using $\mathrm{NaCl} \mathrm{Sam-}$ ples, in Proceedings of the 2016 Symposium on $\mathrm{Nu}$ clear Data; November 17-18, 2016, KEK, Tsukuba, Ibaraki, Japan (2018), pp. 169-174, JAEA-Conf 2017-001

[12] O. Iwamoto, N. Iwamoto, S. Kunieda, F. Minato, K. Shibata, Nuclear Data Sheets 131, 259 (2016)

[13] A. Gilbert, A.G.W. Cameron, Canadian Journal of Physics 43, 1446 (1965)

[14] A. Mengoni, Y. Nakajima, Journal of Nuclear Science and Technology 31, 151 (1994)

[15] R. Capote, M. Herman, P. Obložinský, P. Young, S. Goriely, T. Belgya, A. Ignatyuk, A. Koning, S. Hilaire, V. Plujko et al., Nuclear Data Sheets 110, 3107 (2009)

[16] E.S. Soukhovitski, S. Chiba, O. Iwamoto, K. Shibata, T. Fukahori, G.B. Morogovskij, Tech. rep., Japan Atomic Energy Research Institute (2005), JAERIData/Code 2005-002

[17] J. Kopecky, M. Uhl, Phys. Rev. C 41, 1941 (1990)

[18] IAEA, Tech. rep., IAEA (2006), IAEA-TECDOC1506 